\title{
Health Care Professionals' Perceptions of the Role of Pharmacogenomic Data
}

\author{
WOODIE M. ZACHRY III, PhD, and EDWARD P. ARMSTRONG, PharmD, BCPS, FASHP
}

\begin{abstract}
OBJECTIVE: To explore the perceptions of health care professionals in examining the uses of pharmacogenomic data.

METHODS: A mailed questionnaire elicited respondent perceptions of how the use of pharmacogenomic information would impact the provision of health-related services (7-point scale: $7=$ =strongly increase to $1=$ =strongly decrease). Respondents were also asked to describe their level of agreement to statements related to how pharmacogenomic information should be used (7-point scale: $7=$ strongly agree to $1=$ strongly disagree). One-sample $t$ tests were used to investigate significant differences from the midpoint value of each scale. Survey participants were attendees of a policy conference entitled "Pharmacogenomics: Implications for Patients, Providers, and Payers" sponsored by the university college of pharmacy.
\end{abstract}

RESULTS: Respondents believed the use of pharmacogenomic information would affect several areas of health care, including the cost of insurance premiums $(P<0.001)$, the use of confidential medical information $(P=0.024)$, patient access to therapy $(P=0.005)$, and the impact of physician/patient preferences in selecting treatment choices $(P<0.001)$. Furthermore, respondents felt it should be used to help treat patients $(P<0.001)$, help patients/physicians make therapy choices $(P<0.001)$, create treatment guidelines $(P<0.001)$, conduct research $(P<0.001)$, justify refusals of therapy $(P=0.014)$, and budget for future expenditures $(P<0.001)$. Respondents also believed the information should not be used for setting copay amounts $(P=0.002)$, determining insurance premiums $(P<0.001)$, or in the negotiation of insurance contracts $(P<0.001)$.

CONCLUSION: The respondents to this survey appeared optimistic about the use of pharmacogenomic information, and their responses provided a proactive framework to discuss the potential use and misuse of this technology.

KEYWORDS: Pharmacogenomics, Genetics, Perceptions

J Man Care Pharm 2002:(8)4:278-84

WOODIE M. ZACHRY III, PhD, is Assistant Professor, College of Pharmacy, University of Arizona, Tucson; EDWARD P. ARMSTRONG, PharmD, BCPS, FASHP, is Associate Professor, College of Pharmacy, University of Arizona, Tucson.

AUTHOR CORRESPONDENCE: Woodie M. Zachry III, PhD, Assistant Professor, College of Pharmacy, University of Arizona, 1703 E. Mabel Street, P.O. Box 210207, Tucson, AZ 85721-0207. Tel: (520) 626-5349; Fax: (520) 626-7355; E-mail: zachry@pharmacy.arizona.edu.

Copyright $\odot 2002$ Academy of Managed Care Pharmacy. All rights reserved.
T he realization that vast amounts of pharmacogenomic data may be available in the near future has created both excitement and concern regarding how this information should be used. ${ }^{1-4}$ The topic of pharmacogenomics has become a very popular discussion topic among clinicians, managed care organizations, health policymakers, and academia..$^{5-7}$ Theoretically, the use of pharmacogenomic information will substantially enhance the ability of clinicians to provide individualized drug therapy.-12

Thousands of common genetic variations (e.g. single nucleotide polymorphisms [SNPs]) can influence the likelihood of a patient attaining desired therapeutic outcomes with conventional treatment. ${ }^{13}$ These genetic variations lead to the production of proteins with various degrees of functionality related to drug transport, receptor binding and stimulation, cellular response, metabolism, and excretion. ${ }^{14}$

Polymorphisms have already been identified as determinants of efficacy for beta-agonists (Gly-16 protein), clozapine (5HT2A receptor), and ACE inhibitors. ${ }^{15-17}$ Variations in SNPs can also cause impaired metabolic function. A majority of medications are metabolized by the P450 isoenzymes CYP2D6 and CYP34A. ${ }^{18}$ Impaired drug metabolism in these enzymes can cause an increase in blood concentration of a medication at normal dosages and, subsequently, a higher likelihood of toxic effects. Conversely, impaired CYP function can also reduce the amount of an activated metabolite available for a pharmacological effect. For example, decreased functional capacity of CYP2D6 can translate into a substantially reduced portion of codeine biotransformation to morphine in analgesia. ${ }^{19}$

Use of genotyping technologies may identify targeted populations that can avoid specific adverse drug reactions or reduced effectiveness. ${ }^{4}, 20 \mathrm{~A}$ limited number of academic and teaching hospitals have begun to incorporate pharmacogenomic testing in treatment. Georgetown University provides CYP2D6 profiles, and several companies are developing CYP test kits that should be available within 2 years. ${ }^{21}$ However, the pharmacogenomic revolution will have a substantial cost attributable to new laboratory fees and associated product costs., ${ }^{2,5}$ This may lead to limitations in insurance coverage, or high cost-sharing schemes for consumers. Some applications also raise important ethical questions regarding appropriate use of this technology. ${ }^{22}$ Table 1 provides a list of the potential uses of this technology in managed care settings.

As new pharmacogenomic research is conducted and disseminated, clinicians, managed care organizations, hospitals, governments, and academia will each grapple with many complex and challenging decisions. Substantial discussion is probably needed to determine the optimal ways to incorporate pharmacogenomic data 


\begin{tabular}{|c|c|}
\hline Use & Possible Effects \\
\hline $\begin{array}{l}\text { Development of "gene based" disease } \\
\text { treatment protocols }\end{array}$ & $\begin{array}{l}\text { - Decrease in time to switch to nonpreferred agents in those genetically predisposed to failure } \\
\text { Increase in the immediate use of nonpreferred agents in those genetically predetermined } \\
\text { as high risk for therapeutic failure and/or toxicity with other agents } \\
\text { Increase in the use of concomitant agents for synergistic effects, and/or to decrease } \\
\text { potential for adverse drug events. }\end{array}$ \\
\hline $\begin{array}{l}\text { Pharmacy and health benefits tailored to } \\
\text { meet the needs of the population }\end{array}$ & $\begin{array}{l}\text { - Increased focus on primary prevention in accordance with patient genetic profiles } \\
\text { - Formulary decisions for preferred products influenced by genetic information of population } \\
\text { - Negotiation of risk-sharing arrangements with payers based upon population genetic } \\
\text { information }\end{array}$ \\
\hline $\begin{array}{l}\text { Research for clinically relevant } \\
\text { polymorphisms }\end{array}$ & $\begin{array}{l}\text { - Increased attention to patient confidentiality standards } \\
\text { - Restructuring of drug and food interaction warnings to account for genetic information } \\
\text { - Expanded frontiers for the exploration of the pharmacoeconomic use of medications }\end{array}$ \\
\hline
\end{tabular}

into the coverage decisions and operational procedures in health care organizations. No research has been conducted with health care professionals to determine their perspectives regarding the applications of pharmacogenomic data. Therefore, the purpose of this study was to determine the perceptions of knowledgeable health care professionals in examining the uses of pharmacogenomic data.

\section{Methods}

Data were collected for this study using a 39-item questionnaire developed and pilot-tested by researchers at the University of Arizona. The instrument was divided into 3 sections. The first section asked respondents to indicate how they thought the use of information obtained from pharmacogenomic testing would affect 13 different health care-related items. These items ranged from the impact on patients' access to drug treatments to patients' control over confidential medical information. In this section, respondents were asked to rate the impact of using pharmacogenomic data as one of the following: strongly increase, increase, mildly increase, neither increase nor decrease, mildly decrease, decrease, or strongly decrease. The second section on the questionnaire asked respondents to provide their level of agreement to 21 statements. These statements were related to how information obtained from pharmacogenomic testing should be used. The statements in the second section were items such as "to help determine a medica- tion's place in therapy" and "to help insurance companies forecast and budget." The respondents were asked to rate their responses in the second section as one of the following: strongly agree, agree, mildly agree, neither agree nor disagree, mildly disagree, disagree, or strongly disagree. The third and final section of the questionnaire contained items about the respondents' demographic characteristics. Tables 2 and 3 summarize the responses from the first 2 sections of the survey.

The survey was conducted after gaining approval from the University of Arizona Human Subjects Protection Program. Information about the respondents' voluntary participation was provided in a cover letter that was distributed with the survey. No rewards were offered or provided for completing the questionnaire.

\section{Sample and Design}

In January of every year, the university provides a pharmaceuticalrelated conference for professionals in various facets of the health care industry. Typically, one-third of the audience is from managed care organizations or are health care providers, one-third is from colleges of pharmacy, and one-third is from the pharmaceutical industry. These conferences focus on timely themes dictated by current developments in the health care market. The conference held in January 2001 was titled Pharmacogenomics: Implications for Patients, Providers, and Payers. This conference was intended to stimulate thought and discussion about the possible health 


\section{(TABLE 2 Test Statistics, Means, and Confidence Intervals for the Items Eliciting Respondents' Perceptions} of What Information Obtained from Pharmacogenomic Testing Will Affect

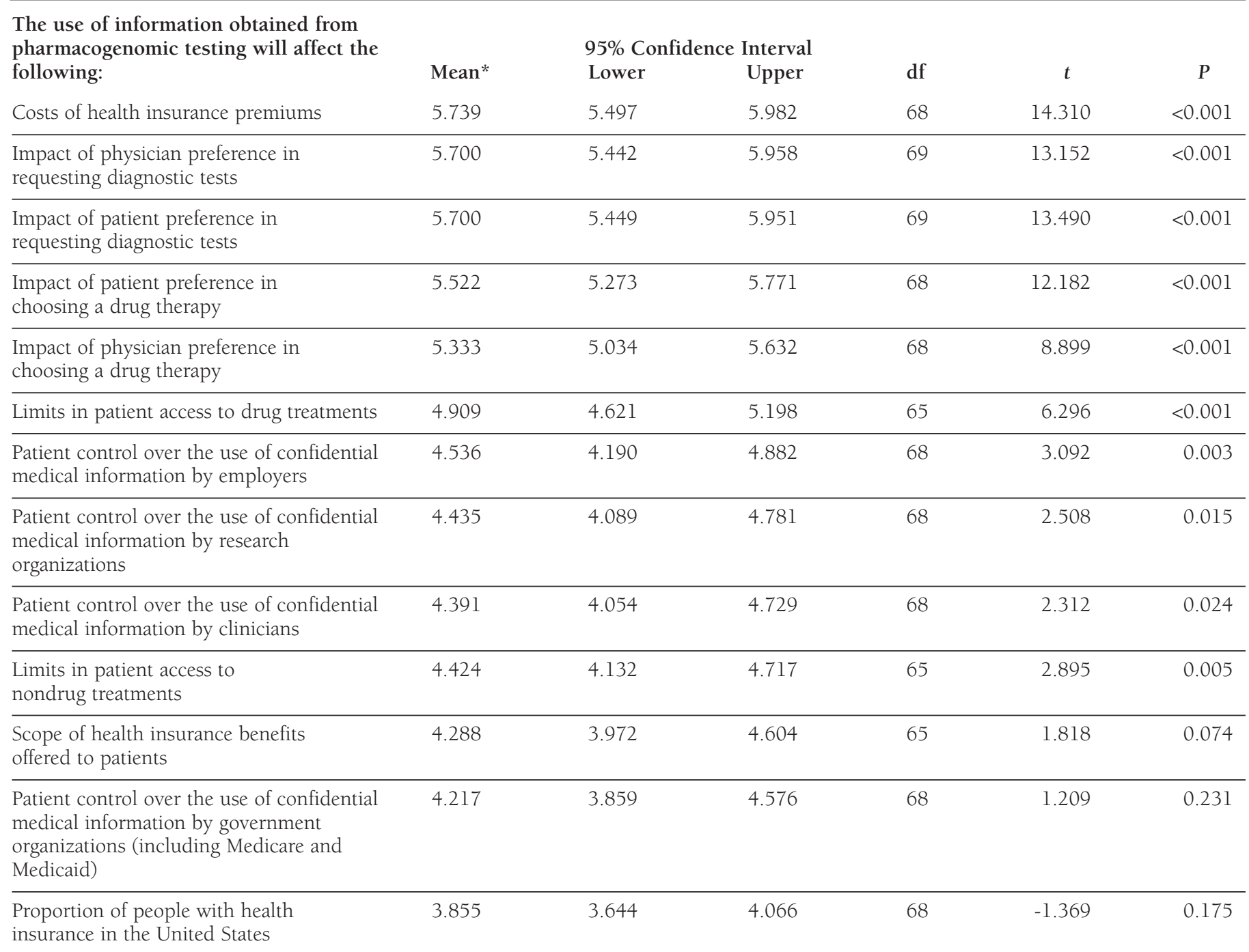

* Responses were given on a 7-point scale:

$7=$ strongly increase, $6=$ increase, $5=$ mildly increase, 4=neither increase nor decrease, $3=$ mildly decrease, $2=$ decrease, $1=$ strongly decrease

care-related implications of pharmacogenomically derived technologies. Several experts from various backgrounds were invited to present and discuss the subject matter over 2 days, with a keynote address provided by George Poste, D.V.M., Ph.D., CEO of Health Technology Networks. Various studies and applications of pharmacogenomic data were presented at the conference.

The attendees of this conference were the focus of the questionnaire. It was believed that these attendees were opinion leaders who represented a population that had sufficient background in the subject area to provide informed opinions concerning the role of pharmacogenomic information. A mail survey design incorporating the methods developed by Don A. Dillman was used to gather responses. ${ }^{23}$ Approximately 11 weeks after the conference, a postcard was mailed to the 87 attendees indicating they would receive a questionnaire the following week. The questionnaire was then mailed with a cover letter and pencil. One week after the questionnaire was mailed, a reminder letter was sent to those who had not responded. Three weeks after mailing the initial questionnaire, a second cover letter, pencil, and questionnaire were sent to the nonresponders.

\section{Statistical Analyses}

The items in sections 1 and 2 were coded on a 7-point interval scale: 7 represented the most positive statement ("strongly increase" 


\section{TABLE 3 Test Statistics, Means, and Confidence Intervals for the Items Eliciting Respondents' Perceptions} of What Information Obtained from Pharmacogenomic Testing Should Be Used For

Information obtained from pharmaco-

genomic testing should be used to

help the following:

Identify patients who will respond

95\% Confidence Interval

to a medication

Identify patients who are at high-risk for

adverse drug events

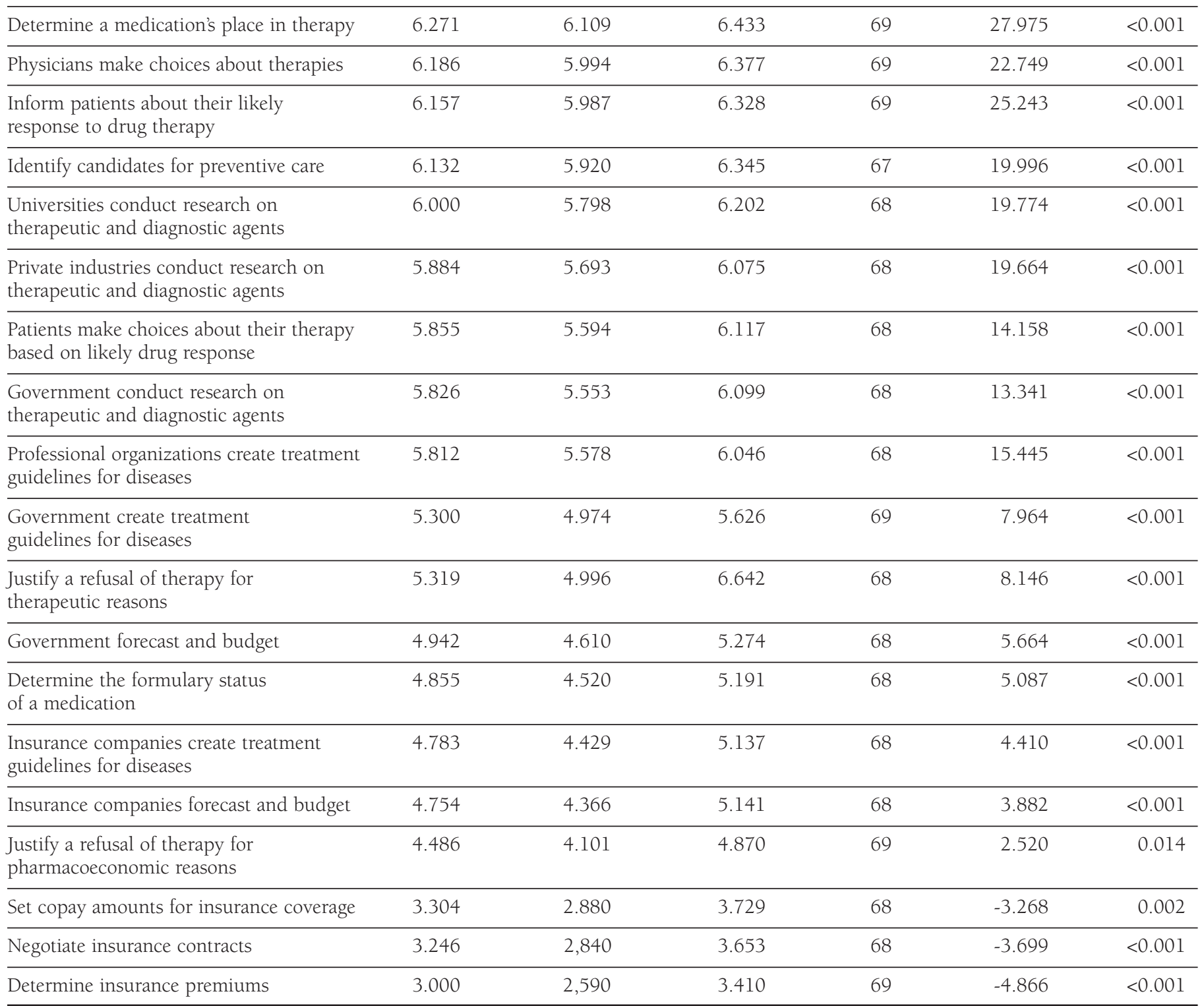

* Responses were given on a 7-point scale:

$7=$ strongly agree, 6=agree, 5=mildly agree, 4=neither agree nor disagree, 3=mildly disagree, 2=disagree, 1=strongly disagree. 
or "strongly agree") decreasing to 1 representing the most negative statement ("strongly decrease" or "strongly disagree"): 4 was considered the midpoint for both scales ("neither increase nor decrease" or "neither agree nor disagree"). One-sample $t$ tests were used to test the hypothesis that scores for each item were not significantly different from the midpoint value of 4 . An a priori alpha level of 0.05 was used for all analyses.

\section{Results}

Only one of the surveys was returned undeliverable. This subject's new address was obtained, and another questionnaire was sent to the new address; the subject responded before the scheduled second mailing of the questionnaire. A $63.2 \%(n=55)$ response rate was obtained by the date of the second mailing of the questionnaire. A total of 17 more subjects responded within 6 weeks after the second questionnaire mailing. Two questionnaires were returned without useable responses. An overall response rate of $80.5 \%$ (70/87) for useable responses was achieved.

Overall, $71.4 \%$ of respondents reported they were male, and $72.9 \%$ of respondents reported they were married. A majority of the respondents reported that an immediate family member suffered from a chronic disease $(60.9 \%$ ), while $24.6 \%$ reported that they themselves suffered from a chronic disease. The mean age of the respondents was 43.4 years old, with a range of 25 to 65 . The respondents worked in a broad range of settings. The largest number of respondents were in an academic setting (38.6\%), followed by the pharmaceutical industry (21.4\%), the insurance and pharmaceutical benefits industry (18.6\%), a clinical practice setting (4.3\%), and a clinical research organization (4.3\%). The remaining respondents (12.9\%) reported that they worked in a setting not listed as one of the choices provided on the questionnaire.

Overall, the results from the first section of the questionnaire suggest that the subjects believed the use of information obtained from pharmacogenomic testing would affect many facets of health care. Respondents felt the use of pharmacogenomic information would increase the impact of patient preference in choosing a drug therapy $(P<0.001)$ and requesting diagnostic tests $(P<0.001)$. Respondents also felt the impact of physician preference in choosing a drug therapy $(P<0.001)$ and requesting diagnostic tests $(P<0.001)$ would increase. Furthermore, respondents believed that patient control over the use of confidential medical information by clinicians $(P=0.024)$, employers $(P=0.003)$, and research organizations $(P=0.015)$ would increase. However, respondents also believed there would be limits in patient access to drug treatments $(P<0.001)$ and nondrug therapies $(P=0.005)$ and that the costs of health insurance premiums would increase $(P=0.005)$.

The results did not support a respondent belief that the use of pharmacogenomic information would affect the proportion of people with health insurance in the United States, the scope of health insurance benefits offered to patients, or patients' control over confidential medical information by government organiza- tions (e.g., Medicare and Medicaid). The results from the first section of the questionnaire are summarized in Table 2.

In the second section of the questionnaire, respondents reported whether they thought information obtained from pharmacogenomic testing should be used for various purposes. Respondents agreed that pharmacogenomic information should be used to determine a medication's place in therapy $(P<0.001)$ and to help government $(P<0.001)$, professional organizations $(P<0.001)$, and insurance companies $(P<0.001)$ create treatment guidelines for diseases. Respondents also agreed that pharmacogenomic information should be used to help physicians $(P<0.001)$ and patients $(P<0.001)$ make choices about therapies. Furthermore, respondents agreed that the information should be used to identify patients who will respond to medications $(P<0.001)$, who are at high risk for adverse events $(P<0.001)$, and who are candidates for preventive care $(P<0.001)$. Respondents also agreed that following pharmacogenomic testing, patients should be informed of their likely response to therapy $(P<0.001)$.

However, the responses were mixed about the use of pharmacogenomic information by insurance companies. On one hand, respondents agreed that the information obtained from pharmacogenomic testing should be used to determine the formulary status of a medication $(P<0.001)$ and to justify a refusal of therapy for pharmacoeconomic $(P=0.014)$ or therapeutic $(P<0.001)$ reasons. On the other hand, respondents did not agree that the information should be used to help determine insurance premiums $(P<0.001)$, negotiate insurance contracts $(P=<0.001)$, or set copay amounts for insurance coverage $(P=0.002)$. However, respondents did agree that pharmacogenomic information should be used to help both the insurance industry $(P<0.001)$ and the government $(P<0.001)$ forecast and budget.

Finally, respondents were receptive to the idea that information derived from pharmacogenomic testing should be used to further research. Respondents agreed that the information should be used to help universities $(P<0.001)$, private industries $(P<0.001)$, and government $(P<0.001)$ conduct research on therapeutic and diagnostic agents. The results from the second section of the questionnaire are summarized in Table 3.

\section{Discussion}

The results of this survey are important in that they outline the opinions of a sample of professionals who may be in a position to influence or make treatment and policy decisions. The sample frame of attendees included many people in leadership roles in their respective organizations. Among the titles listed by academic attendees, 4 were deans or assistant deans, 3 were full professors, 9 were associate professors, and 2 were assistant professors. Among the titles listed by attendees employed in the private sector, 7 were presidents or equivalent, 6 were vice presidents, 17 were directors, and 10 were managers. The remaining attendees listed titles including clinical pharmacist, researcher, scientist, 
economist, publisher, coordinator, student, and liaison. These results provide a proactive examination of the potential impact of issues associated with pharmacogenomic testing. The data represent informed opinions from professionals about how pharmacogenomic information will—and should-be used. These respondents believed that information obtained from pharmacogenomic testing will have a far-reaching influence in the future provision of health care in the United States.

Overall, the respondents felt the use of this information will alter the roles of patients and physicians, impact the use of confidential medical information, and change the economics of health care insurance. In general, respondents appeared to be cautiously optimistic about the use of pharmacogenomic information. Respondents believed the use of pharmacogenomic information would increase the impact of physician and patient preference in the provision of care (i.e., treatment and diagnostic test requests) but would also result in new limits in patient access to certain treatment options. Although the use of pharmacogenomic information might be used to justify the use of nonpreferred agents in those patients with a demonstrated need, this information might also be used to restrict use in those patients with a low probability of success with a nonpreferred agent.

Respondents believed that the use of pharmacogenomic information would be associated with increases in patient and physician control over confidential information. However, the respondents also believed the use of this information would be associated with increases in the cost of insurance premiums, though they did not think the data should be used in making economic decisions (e.g., setting premiums, copays). On the other hand, respondents were supportive of the use of the information for budget forecasting. Finally, respondents supported the use of pharmacogenomic information for therapeutic and research goals and for justifying refusals of therapy.

No other studies were identified in the literature with which to compare the findings of the present study. Pharmacogenomics is a growth area and too new for widespread applications by existing health care professionals. ${ }^{4}$ Future research is needed to clarify the perceptions of health care professionals as pharmacogenomic techniques become more widely used. Pharmacogenomics could dramatically affect the provision of prescription drug benefits over the next 5 to 10 years. Some predict the majority of drugs newly approved by the FDA in 5 to 10 years will be intravenous (IV) formulations, and $80 \%$ of drugs now in solid dosage form will become available as IV fluids. ${ }^{24}$ Genetic testing that fragments a viable market may even create disincentives for pharmaceutical research and development in some subsets of the population. ${ }^{25}$ From a managed care perspective, many observers also predict increased use of laboratory tests and genetic counseling. ${ }^{26}$

Access to genomic information offers the opportunity to reduce the incidence of trial-and-error in selecting optimum drug therapy for individual patients. Genomic information may also permit exclusion of patients who will not respond adequately to a particular drug therapy before exposing the patient to the drug. Also, drug therapy will be targeted more precisely by drug type and dose to the individual patient, reducing the opportunity for untoward side effects. As more genomic information is made available, clinicians will likely have to follow more stringent prescribing protocols, especially when the risk to the patient is high. ${ }^{27}$ The application of this technology could herald a more expensive future for pharmaceutical products on a per-use basis, but this higher cost per patient could be offset by reducing the number of patients exposed to ineffective and/or harmful treatments.

\section{Limitations}

There are several important limitations to this study. First, the sample in the study represents participants in a conference concerning a very new topic. Substantial new research may be forthcoming that will significantly alter the perceptions of the respondents. Second, although the conference provided a broad education by pharmacogenomic experts, it is possible that biases were introduced that influenced the perceptions of the respondents. Third, although the conference participants represented a wide range of health care and health policy professionals, the respondents may not fully reflect the viewpoints of professionals throughout the United States. Finally, 17 people did not respond to the questionnaire. It is possible that the addition of their responses could have added to the generalizability of the results.

\section{Conclusions}

The realization that pharmacogenomic data may be available in the near future has raised many complex questions regarding the optimal use of diagnostic and therapeutic agents. Although still preliminary, application of this information may be especially useful to better target the use of innovative products. Respondents in this study were optimistic regarding the theoretical benefits of identifying patient populations most likely to achieve optimal therapeutic outcomes. However, future research and transparent discussion are needed to ethically and therapeutically incorporate these data into health care decisions.

\section{DISCLOSURES}

This study and education program were supported by an unrestricted educational grant from the Merck Company Foundation, the philanthropic arm of Merck \& Company, Inc.; funding was obtained by authors Woodie M. Zachry III and Edward P. Armstrong. Neither author has financial interests or is affiliated with any company or product mentioned in this article.

Zachry served as principal author of the study. Study concept and design, analysis and interpretation of data, drafting of the manuscript, and critical revision of the manuscript was conducted jointly and equally by the authors; statistical expertise was contributed by both authors. 


\section{REFERENCES}

1. Carrico JM. Human Genome Project and pharmacogenomics-implications for pharmacy. J Am Pharm Assoc. 2000;40:115-16.

2. Peterson C. Genomics and managed care: preparing for the revolution. Healthplan. 2000;41:14-20.

3. Becker C. The DNA Rx. Advances in genetics give physicians ability to tailor drugs to patients' unique makeup. Mod Healthcare. 2000;30:24-28,30,33

4. Rusnak JM, Kisabeth RM, Herbert DP, McNeil DM. Pharmacogenomics: a clinician's primer on emerging technologies for improved patient care. Mayo Clin Proc. 2001;76:299-309.

5. Swartz K. The human genome and medical care in the new century. Inquiry. 2000;37:3-6.

6. Rioux P. Clinical trials in pharmacogenetics and pharmacogenomics: methodology and applications. Am J Health-Syst Pharm. 2000;57:887-98.

7. Evans WE, Relling MV. Pharmacogenomics: translating functional genomics into rational therapeutics. Science. 1999;286:487-91.

8. Sadee W. Pharmacogenomics. Brit Med J. 1999;319:1-4.

9. Nebert DW. Pharmacogenetics and pharmacogenomics: why are they relevant to the clinical geneticist? Clin Genet. 1999;56:247-58.

10. Murphy MP. Current pharmacogenomic approaches to clinical drug development. Pharmacogenomics. 2000;1:115-23.

11. Adam GI, Reneland R, Andersson M, Risinger C, Nilsson M, Lewander T. Pharmacogenomics to predict drug response. Pharmacogenomics. 2000;1:514.

12. Vesell ES. Advances in pharmacogenetics and pharmacogenomics. J Clin Pharmacol. 2000;40:930-38

13. Langevin BC. Pharmacogenomics: tailoring drug therapy. J Am Pharm Assoc. 1999;39:597-98.

14. Freeman TR. Pharmacogenomics: opening new vistas in pharmacotherapy. J Am Pharm Assoc. 2001;41:629-30.
15. Martinez FD, Graves PE, Baldini M, et al. Association between genetic polymorphisms of the beta2-adrenoceptor and response to albuterol in children with and without a history of wheezing. J Clin Invest. 1997;100:3184-88.

16. Nakano Y, Oshima T, Wantanabe M, et al. Angiotensin I-converting enzyme gene polymorphism and acute response to captopril in essential hypertension. Am J Hypertens. 1997;10:1064-68.

17. Arranz MJ, Munro J, Sham P, et al. Meta-analysis of studies on genetic variation in 5-HT2A receptors and clozapine response. Schizophr Res. 1998;32:93-99.

18. Riox PP. Clinical trials in pharmacogenetics and pharmacogenomics: methods and applications. Am J Health-Syst Pharm. 2000;57:887-98.

19. Desmeules J, Gascon MP, Dayer P, Magistris M. Impact of environmental and genetic factors on codeine analgesia. Eur J Clin Pharmacol. 1991;41:23-26.

20. Phillips KA, Veenstra DL, Oren E, Lee JK, Sadee W. Potential role of Pharmacogenomics in reducing adverse drug reactions. JAMA. 2001;286:2270-79.

21. Norton RM. Pharmacogenomics and individualized drug therapy. Available at: http://www.medscape.com/medscape/pharmacology/journal/2001/mp7520.nort.html. Accessed January 17, 2002.

22. Issa AM. Ethical considerations in clinical pharmacogenomics research. Trend Pharmacol Sci. 2000;21:247-49.

23. Dillman DA. Mail and telephone surveys: The Total Design Method. New York, New York: John Wiley \& Sons; 1978.

24. Levy S. How genomics will impact pharmacy. Drug Top. 2002;146:24.

25. Danzon P, Towse A. The economics of gene therapy and of pharmacogenetics. Value in Health. 2002;5:5-13.

26. Veenstra DL. Pharmacoeconomics and pharmacogenomics: the end of blockbuster drugs? ISPOR News. 2001;7:10.

27. Higashi MK, Veestra DL, Kondo LM, et al. Association between CYP2C9 genetic variants and anticoagulation-related outcomes during warfarin therapy JAMA. 2002;287:1690-98. 